\title{
Swarming of Mobile Router Networks
}

\author{
Nicola Bezzo and Rafael Fierro
}

\begin{abstract}
A decentralized method for mobile router networks is proposed in this paper. A group of mobile routers moves in a cluttered environment maintaining connectivity between a base station and multiple exploring users. The network is seen as a switched system in which virtual springmass interactions create and destroy the links between agents. We prove static (i.e., fixed graph topology) and dynamic (i.e., switching graph topology) stability using Lyapunov theory. To demonstrate the applicability of this method, we offer simulations for a self-healing case and for a non-isotropic environment.
\end{abstract}

\section{INTRODUCTION}

Communication in robotics is a recent topic that has raised the attention of the control community both because of the challenges that wireless communication offers and because real world applications require a stable and reliable communication infrastructure. This is especially true during delicate operations such as homeland security, disaster relief operations and search and rescue missions (Fig. 1).

Radio communication is uncertain and is characterized by randomness because every environment is different and the presence of obstacles, both fixed and mobile, affects the quality of the propagated and received signal.

Modern modems and routers are very compact and small (e.g., the Zigbee module). Most of them are characterized by small power transmission and bandwidth (i.e., power supplied by usb ports), noisy signal, and interference that affect the overall quality of the transmission. Nevertheless, these devices have limited range of operation and the signal decays following the typical path loss model found in the literature, [1]. In order to keep communication as a constraint, some typical parameters are the Signal to Noise Ratio (SNR), the Capacity of the channel, the Signal Strength, and the Bit Error Rate (BER), [1].

In our previous work [2], we presented a framework based on disjunctive programming to tether a chain of mobile routers and keep a base station connected to a user that explores an unknown environment. This method allows to build a chain of robots that adapts and stretches while the user moves. However this method is centralized, making it less general and computationally complex: all decisions are made by a central controller that has to compute cluttered environments populated with multiple robots.

In this paper we evaluate the same type of problem in which the goal is to maintain a base station connected with some users that move in an environment populated with obstacles. With this work we are able to have a distributed scenario in which all mobile routers make decisions based on their neighbors and, at the same time, we extend our previous work using multiple users.

N. Bezzo and R. Fierro are with the MARHES Lab, Department of Electrical and Computer Engineering, University of New Mexico, Albuquerque, NM 87131-0001, USA \{nicbezzo, rfierro\}@ece.unm.edu

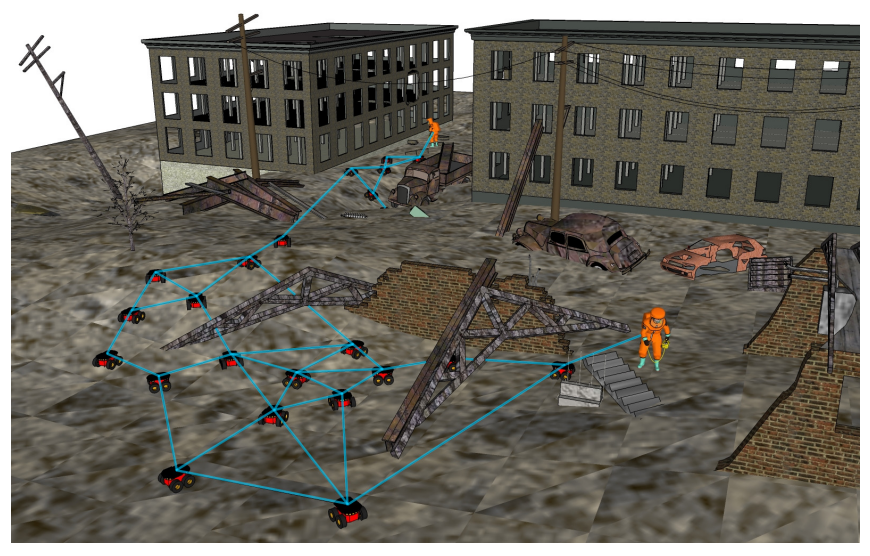

Fig. 1. A virtual environment with mobile routers. The lines between the robots represent sensed connectivity paths.

\section{A. Related Work}

Communications and robotics have received a lot of attention in the last couple of years, especially after the LANdroid program [3]. Some of the major conferences around the world now host special sessions to cover this topic in order to have a more heterogeneous and realistic scenario, [4], [5], [6], [7].

Modern wireless communications have been around for more than twenty years [8]. Even though there has been progress in recent devices, many problems and uncertainties still exist. Shadowing, multipath, and scattering are the major disturbances when dealing with communication through air. Because of the nature of the wireless infrastructure, we need to deal with randomness. Many groups in the community have offered solutions, with different degrees of complexity, to make wireless more robust and reliable.

Similarly to our previous work, the authors in [9] propose a geometric type approach to maintain a single user connected with a base station in a simply connected polygonal type environment with circular obstacles.

Authors in [6] present a method based on maximum likelihood estimation to estimate the position of a radio source in an unknown environment, therefore giving a mapping of the signal and an estimate of the position of the source. Similar to this approach is the work in [10] where the authors describe a motion planning algorithm in which a robot, by making a few measurements, is able to learn and predict the link quality with a fixed base station.

A stop-time policy is proposed in [4]: a mobile router moves following a specific trajectory and regularly stops and measures the signal strength, moving faster where the signal is poor and staying longer where the capacity is high.

The use of aerial vehicles is found in [7] where UAVs act as relays to build the communication infrastructure for a team of ground vehicles. The authors design a gradient based controller and incorporate the Signal to Interference Ratio 
(SIR) taking into account communication as a constraint. It is demonstrated that given appropriate initial conditions, the controller is able to drive the aerial vehicles to optimal positions that guarantee connectivity between the ground robots without disconnections.

Standing more from a control point of view are the works in [11] [12] [13]. In [11] the authors explore graph connectivity for a distributed control of a mesh of robots by using local estimates of the network. Gossip algorithms and distributed market-based control are used to break connection while potential field is used to keep exiting links connected to the network. A proof of stability in the sense of connectivity is offered by using second smallest eigenvalue together with the notion of Laplacian, [14].

Graph theory is also used in [12] to keep a group of robotic explorers connected using potential functions.

Finally authors in [13] use a spring-mass system together with graph theory in order to demonstrate stability of a swarm or robots that move in a decentralized fashion to cover an area while maintaining connectivity.

Our work is motivated by the scheme developed in [13]. However we consider a connectivity problem using mobile routers. Specifically we build a spring-mass system in which sensing and connectivity among agents are represented by virtual links, that are created and removed according to specific switching rules, and communications constraints are represented by potential functions.

This paper is organized as follows. In Section II we present the robot, obstacle and communication models. In Section III there is a detailed description of the constraints followed by the algorithm in Section IV . In Section V we show simulation results and finally, conclusions are drawn in Section VI .

\section{PRELIMINARIES}

In this section we present the theory and tools that are behind the implementation of the decentralized controller, which is described in more details in Section III.

\section{A. Robot model}

In our paper the mesh of robots is built following the spring-mass virtual physic. We base our model from the work in [13] where the robots are treated as point-mass particles. Similar work can be found in [15] where the authors treat the robots like the particles that move in a gas.

The spring-mass approach in this paper produces a uniform deployment of the robots in a closed environment and, as it will be demonstrated, we reach always a situation of equilibrium.

The dynamics of the $i^{\text {th }}$ spring-mass router, among the total $\mathcal{N}_{r}$ available, follows the model:

$$
\begin{gathered}
\ddot{X}_{i}=\left[\sum_{j \in \mathcal{S}_{i}} k_{i j}\left({ }_{i j}-l_{i j}^{0}\right) \hat{\mathbf{d}}_{i j}\right]-\gamma_{i} \dot{X}_{i}, \\
\text { with } i=1, \ldots, \mathcal{N}_{r} \text { and } i \neq j,
\end{gathered}
$$

where $X_{i}=\left(x_{i}, y_{i}\right)^{T}$ is the position vector of each robot relative to a fixed Euclidean frame, and $\dot{X}_{i}, \ddot{X}_{i}$ denote the velocity and acceleration (control input), respectively. $\mathcal{S}_{i}$ is the set of neighbor robots connected to the $i^{\text {th }}$ router and since we are using a spring-mass model, these links between agents are virtual springs; $l_{i j}$ is the length of the spring between robot $i$ and $j$ and $l_{i j}^{0}$ is the rest spring length; $\hat{\mathbf{d}}_{i j}$ is the unit vector indicating the direction of the force of the virtual spring between the robots and finally $k_{i j}$ and $\gamma_{i}$ are the spring constant between robots $i$ and $j$ and the damping coefficient, respectively. We assume $\gamma_{i}>0$.

Since we take into consideration damping effects, the mesh of virtual springs has similar behavior as a real spring system in which dissipative forces act against the movement of the springs ensuring velocity to eventually reach zero.

\section{B. Obstacle and collision avoidance}

In order to prevent collisions with obstacles we define a workspace repulsive potential field, [16]. A common way to proceed is to build a potential whose value approaches infinity as the robot approaches the obstacle, and whose value goes to zero if the robot is at a distance greater than $\rho_{0}$ from the obstacle. Formally:

$$
\begin{aligned}
& W_{O, i}=\left\{\begin{array}{ll}
\frac{1}{2} \eta_{i}\left(\frac{1}{\rho\left(X_{i}\right)}-\frac{1}{\rho_{0}}\right)^{2} & \text { if } \rho\left(X_{i}\right) \leq \rho_{0} \\
0 & \text { if } \rho\left(X_{i}\right)>\rho_{0}
\end{array},\right. \\
& \text { with } i=1, \ldots, \mathcal{N}_{r} \text {, }
\end{aligned}
$$

where $\rho\left(X_{i}\right)$ is the shortest distance between $X_{i}$ and any obstacle in the workspace and $\eta_{i}$ is a positive constant.

Then, the repulsive force is equal to the negative gradient of $W_{O, i}$, or in formula for $\rho\left(X_{i}\right) \leq \rho_{0}$, it is given by:

$$
F_{O, i}=\eta_{i}\left(\frac{1}{\rho\left(X_{i}\right)}-\frac{1}{\rho_{0}}\right) \frac{1}{\rho\left(X_{i}\right)^{2}} \nabla \rho\left(X_{i}\right),
$$

where $\nabla \rho\left(X_{i}\right)$ is the gradient of the minimum distance between the $i^{\text {th }}$ robot and the closest obstacle. It is essential to note that the expression in (3) exists only if (2) is differentiable. For a polygon type obstacle, (2) is not differentiable everywhere but we can think of the obstacle as covered by set of disks. Thus, in this way we can always find the gradient of the minimum distance between robots and obstacles.

Trivially, the forces created by the virtual springs between the robots allow collision avoidance at every time.

\section{Communication model}

In this work we use a line-of-sight approach, meaning that we assume communication between two nodes only if the nodes have a clear view of each other. We also consider that in the environment there are $\mathcal{N}_{p}$ patches of areas in which the signal quality is below a certain threshold. The motivation for this choice is as follows.

The majority of the literature in robotics and communication considers just a simple path loss model for wireless. However there are other two significant modes that affects the quality of the signal propagated from a transmitter to a receiver: multipath and fading, [1], [17]. Both these characteristics depend on the fact that the signal is obstructed, reflected, scattered by the presence of obstacles such as people, walls, hardware and buildings. Therefore to consider these effects, we create some repulsive potential functions so that the robots are attracted to areas in the environment where the quality of the signal is better.

For simplicity sake we assume a multi repulsive function of the form:

$$
\nu\left(X_{i}\right)=\sum_{j=1}^{\mathcal{N}_{p}} \frac{A_{\nu}^{j}}{2} e^{-\frac{\left\|X_{i}-c_{\nu}^{j}\right\|^{2}}{\ell_{\nu}^{j}}},
$$


where $A_{\nu}^{j} \in \mathbb{R}^{+}, \ell_{\nu}^{j} \in \mathbb{R}^{+}, c_{\nu}^{j} \in \mathbb{R}^{2}$. The gradient of (4) is:

$$
\nabla_{X_{i}} \nu\left(X_{i}\right)=-\sum_{j=1}^{\mathcal{N}_{p}} \frac{A_{\nu}^{j}}{\ell_{\nu}^{j}}\left(X_{i}-c_{\nu}^{j}\right) e^{-\frac{\left\|X_{i}-c_{\nu}^{j}\right\|^{2}}{\ell_{\nu}^{j}}} .
$$

\section{COORDinAtion OF MOBILE Routers Via SWITCHED SySTEMS}

In this work we are interested in the problem of maintaining connectivity between a base station $b$ and some users $\mathcal{T}_{j}$ that move within an obstacle-populated environment.

Clusters of robots are available to achieve this goal covering the area of interest avoiding obstacles and collision in a fully decentralized fashion.

\section{A. Problem Formulation}

Formally the swarming connectivity problem can be state as follows:

Problem 3.1: Controlled connectivity using mobile router swarms: Given $M$ sets $\mathcal{M}_{M}$ of $\mathcal{N}_{r, M}$ mobile routers and $\mathcal{T}_{j}$ with $j \in \mathbb{N}$ users moving within a specified area $\mathcal{W}$ with an unknown trajectory $g_{\mathcal{T}}(t) \in \mathbb{R}^{2}$, find a set of feasible policies $\mathbf{u}_{i} \in \mathcal{U}$ for each router such that the users are connected all of the time to the fixed base station $b$, once a connection is established.

Fig. 1 shows an example of the situation we are describing in problem 3.1 in which a swarm of robots covers an area populated with obstacles and at the same time keeps connectivity with a couple of exploring users.

\section{B. Gabriel Graph}

By using the spring-mass model (1) we can build a mesh or robots that doesn't expand and cover well the environment. Therefore to overcome this limitation and thus have a more complete coverage of the workspace, we add the following constraints.

1) Between any two robots $i$ and $j$, we form a spring if and only if there is no robot $k$ inside the circle of diameter $\overline{i j}$, [18]. The same constraint applies between robots and base station and between robots and users. We define a variable $\tau$ that is 1 if there is a spring between two robots and 0 vice versa. Formally the constraint is represented as follows:

$$
\tau_{i j}=\left\{\begin{array}{ll}
1 & \text { if } \widehat{i k j} \leq \pi / 2 \\
0 & \text { if } \widehat{i k j}>\pi / 2
\end{array},\right.
$$

with $i, j, k=1, \ldots, \mathcal{N}_{r}$ and $i \neq j \neq k$,

where $\widehat{i k j}$ is the interior angle of the three robots configuration.

2) For a more general case we assume that in the environment there are clusters of robots, with each cluster located far apart from one another. Therefore the robots inside every cluster can communicate with each other but they cannot communicate to the routers of another cluster unless they get close enough to guarantee connectivity.

Between clusters of robots we assume we can form at least a spring following rule (6) and limiting the communication range to a threshold equal to $\varphi$. In other words mathematically we can express this constraint as follows:

$$
\begin{gathered}
\delta_{i l}=\left\{\begin{array}{ll}
1 & \text { if }\left\|X_{i}-X_{l}\right\| \leq \varphi \\
0 & \text { if }\left\|X_{i}-X_{l}\right\|>\varphi
\end{array},\right. \\
\text { with } i \in \mathcal{M}_{i} \text { and } l \in \mathcal{M}_{l},
\end{gathered}
$$

where $\mathcal{M}_{i}$ and $\mathcal{M}_{l}$ are two distinctive clusters of robots with $\left\{\mathcal{M}_{i}, \mathcal{M}_{l}\right\} \in \mathcal{M}_{M}$, and $\delta$ is a binary variable that is equal to 1 if the $i^{\text {th }}$ mobile router $\in \mathcal{M}_{i}$ detects the $l^{\text {th }}$ mobile router $\in \mathcal{M}_{l}$.

$\tau_{i j}$ and $\delta_{i l}$ affect the value of $\mathcal{S}_{i}$ in (1). Therefore given (6) and (7), the control law in (1) can be rewritten as:

$$
\begin{aligned}
\ddot{X}_{i} & =\left[\sum_{j=1}^{\mathcal{N}_{r, i}} \tau_{i j} k_{i j}\left(l_{i j}-l_{i j}^{0}\right) \hat{\mathbf{d}}_{i j}\right] \\
& +\sum_{m=1}^{M}\left[\sum_{l \in \mathcal{M}_{m}} \delta_{i l} k_{i l}\left(l_{i l}-l_{i l}^{0}\right) \hat{\mathbf{d}}_{i l}\right]-\gamma_{i} \dot{X}_{i}, \\
& \text { with } i=1, \ldots, \mathcal{N}_{r, i}, i \neq j \text { and } i \notin \mathcal{M}_{m},
\end{aligned}
$$

where $\mathcal{N}_{r, i}$ represents the total number of robots inside the cluster where the $i^{\text {th }}$ robot operates and $\mathcal{M}_{m}$ is the $m^{\text {th }}$ cluster of mobile routers among the total $M$.

\section{Controller}

By assembling together all the pieces described in the previous sections, the overall controller is as follows:

$$
\begin{aligned}
\ddot{X}_{i} & =\left[\sum_{j \in \mathcal{S}_{i}} k_{i j}\left(l_{i j}-l_{i j}^{0}\right) \hat{\mathbf{d}}_{i j}\right]-\gamma_{i} \dot{X}_{i} \\
& +\eta_{i}\left(\frac{1}{\rho\left(X_{i}\right)}-\frac{1}{\rho_{0}}\right) \frac{1}{\rho\left(X_{i}\right)^{2}} \nabla \rho\left(X_{i}\right) \\
& -\nabla_{X_{i}} \nu\left(X_{i}\right) .
\end{aligned}
$$

\section{Switched systems: Stability Analysis}

The constraints introduced in Section III-A produce a connectivity graph that is often referred in literature as Gabriel Graph [13], [18]. While the formation of this graph has a lot of practical advantages, the continuous construction and destruction of springs create problems in terms of proving stability. In fact, the spring-mass virtual system represents a switched system since there is a coupling between continuous dynamics and discrete events.

However in the literature, [19], [20], [13], we find a dwell time analysis that provides stability for general cases, by creating restrictions on the switching rate.

Also the work in [21] shows a proof of stability of a similar spring-mass system by imposing bounds on the damping. More specifically it is proven that if $2 \sqrt{k_{i j}} \leq$ $\gamma_{i} \leq \frac{\left|\left(\dot{X}_{i}^{T} \dot{X}_{i}\right) / \Delta t+\sum_{j \in \mathcal{S}} k_{i j}\left(l_{i j}-l_{i j}^{0}\right) \hat{\mathbf{d}}_{i j}\right|}{\left|\dot{X}_{i}^{T} \dot{X}_{i}\right|}$, the system reaches dynamic stability.

In this paper we present a general proof of stability based on [13]. By using an appropriate Lyapunov function and Barbalat's lemma, we prove first static stability and then dynamic stability in an obstacle-populated environment.

Theorem 3.2: The virtual spring-mass system (9) with switching topology reaches a rest state with a constant potential after a certain amount of time.

Proof: Static Stability. The system shown in (9) is stable in absence of switching or if we consider the intervals between the switches.

Let: 


$$
\begin{aligned}
V_{i} & =\frac{1}{2} \sum_{j \in \mathcal{S}_{i}} P_{i j}+\frac{1}{2} \dot{X}_{i}^{T} \dot{X}_{i} \\
& +\frac{1}{2} \eta_{i}\left(\frac{1}{\rho\left(X_{i}\right)}-\frac{1}{\rho_{0}}\right)^{2}+\nu\left(X_{i}\right),
\end{aligned}
$$

be the Lyapunov function associated with our system with $P_{i j}=k_{i j}\left(l_{i j}-l_{i j}^{0}\right)^{2}$. Since all the terms in (10) are positive, $\forall i=1, \ldots, \mathcal{N}_{r}, V_{i}$ is positive definite.

The total energy function for the system is:

$$
V=\sum_{i=1}^{\mathcal{N}_{r}} V_{i}
$$

and clearly it is positive definite.

By taking the first derivative of (11), we obtain:

$$
\begin{aligned}
\frac{d V}{d t} & =\sum_{i=1}^{\mathcal{N}_{r}}\left[\frac{1}{2} \sum_{j \in \mathcal{S}_{i}} \dot{P}_{i j}+\dot{X}_{i}^{T} \ddot{X}_{i}\right] \\
& -\sum_{i=1}^{\mathcal{N}_{r}} \eta_{i}\left(\frac{1}{\rho\left(X_{i}\right)}-\frac{1}{\rho_{0}}\right) \frac{1}{\rho\left(X_{i}\right)^{3}}\left(X_{i}-X_{o}\right)^{T} \dot{X}_{i} \\
& -\sum_{i=1}^{\mathcal{N}_{r}} \sum_{j=1}^{\mathcal{N}_{p}} \frac{A_{\nu}^{j}}{\ell_{\nu}^{j}}\left(X_{i}-c_{\nu}^{j}\right) e^{-\frac{\left\|X_{i}-c_{\nu}^{j}\right\|^{2}}{\ell_{\nu}^{j}}}
\end{aligned}
$$

where $X_{o}$ is the coordinate of the closest point of the nearest obstacle to $X_{i}$.

Now substituting for $\ddot{X}_{i}$ in (12) with the expression in (9), it is easy to see that the derivative in (12) becomes:

$$
\frac{d V}{d t}=-\sum_{i=1}^{\mathcal{N}_{r}}\left(\gamma_{i} \dot{X}_{i}^{T} \dot{X}_{i}\right) .
$$

Since $\gamma_{i}>0$, last term derived in (12) represents the total energy dissipated by damping and it is clearly negative semidefinite.

Finally taking the derivative of (13) we obtain:

$$
\frac{d^{2} V}{d t^{2}}=-2 \sum_{i=1}^{\mathcal{N}_{r}}\left(\gamma_{i} \dot{X}_{i}^{T} \ddot{X}_{i}\right) .
$$

The expression in (14) is finite as long as the mobile routers speed and $\left(l_{i j}-l_{i j}^{0}\right)$ are finite. Therefore, by Barbalat's lemma, the control law in (9) guarantees that the system is stable between switches, [22].

Dynamic Stability. In order to prove dynamic stability for this type of systems we use a similar argument of [22] in which the authors introduce an energy reserve $\Delta E$ to cancel the switching effects and prove stability.

Following this approach, we see that, in an interval of time $\Delta t$ big enough to cover a switch between two topologies, the rate of variation of $V$ is:

$$
\frac{\Delta V}{\Delta t}=\sum_{i=1}^{\mathcal{N}_{r}}\left[\frac{1}{2} \sum_{j \in \Delta \mathcal{S}_{i}} k_{i j}\left(l_{i j}-l_{i j}^{0}\right)^{2}-\gamma_{i}\left(\Delta_{X_{i}}^{t}\right)^{T}\left(\Delta_{X_{i}}^{t}\right)\right] \text {, }
$$

where the first term reflects the changes due to switching in the system and $\Delta_{X_{i}}^{t}=\frac{\Delta X_{i}}{\Delta t}$.

We can build a new potential function $V_{\text {new }}=V+E$ with $E$ solution of the equation:
$\frac{\Delta E}{\Delta t}=\frac{1}{2} \sum_{i=1}^{\mathcal{N}_{r}}\left[\gamma_{i}\left(\Delta_{X_{i}}^{t}\right)^{T}\left(\Delta_{X_{i}}^{t}\right)-\sum_{j \in \Delta \mathcal{S}_{i}} k_{i j}\left(l_{i j}-l_{i j}^{0}\right)^{2}\right]$,

which depends on the changes in $\mathcal{S}$.

Substituting in the new potential function and taking the expression back to a differential form we obtain:

$$
\frac{d V_{\text {new }}}{d t}=\frac{d V}{d t}+\frac{d E}{d t}=-\frac{1}{2} \sum_{i=1}^{\mathcal{N}_{r}}\left(\gamma_{i} \dot{X}_{i}^{T} \dot{X}_{i}\right),
$$

which is negative semi-definite as desired. Finally differentiating (15) we obtain:

$$
\frac{d^{2} V_{n e w}}{d t^{2}}=-\sum_{i=1}^{\mathcal{N}_{r}}\left(\gamma_{i} \dot{X}_{i}^{T} \ddot{X}_{i}\right) .
$$

Therefore the new function has the following properties:

- $V_{\text {new }}$ is positive-definite;

- $\dot{V}_{\text {new }}$ is negative semi-definite;

- $\ddot{V}_{\text {new }}$ is bounded and finite.

By Barbalat's lemma we conclude that the system with the new energy function is also dynamically stable.

\section{E. Decentralized Deployment}

By assembling together everything we described in the previous sections, the overall problem is decentralized because every robot, by sensing its neighbors, has to compute the spring-mass algorithm in (9) subject to: the constraint on the angle (6) and the constraint on the clusters (7).

Together with these constraints we have to make sure that the robots are not in singular positions, for example, perfectly aligned with one another. In such singular positions the forces created by the virtual springs keep the routers aligned in rows and avoid the complete coverage of the environment.

The constraint associated with this problem is:

$$
\begin{gathered}
\widehat{i j k}=\pi+\omega \quad \text { if } \quad \widehat{i j k}=\pi, \\
\text { with } i, j, k=1, \ldots, \mathcal{N}_{r} \text { and } i \neq j \neq k,
\end{gathered}
$$

where $\widehat{i j k}$ is the inner angle of the configuration made of three robots of the same cluster and $\omega$ is a perturbation added to the angle in order to avoid to have virtual forces aligned on the same axis.

If the users, mobile routers and base station are connected, we can guarantee connectivity at all time if and only if:

$$
\begin{gathered}
\dot{X}_{\mathcal{T}_{j}} \leq \dot{X}_{i}, \\
\text { with } j=1, \ldots, \mathcal{N}_{\mathcal{T}} \text { and } i=1, \ldots, \mathcal{N}_{r},
\end{gathered}
$$

where $\dot{X}_{\mathcal{T}_{j}}$ is the velocity of the $j^{\text {th }}$ user among the total $\mathcal{N}_{\mathcal{T}}$ users and $\dot{X}_{i}$ is the maximum velocity of the $i^{\text {th }}$ mobile router connected with that specific user.

On the other hand if the presence of obstacles and large distances does not guarantee connectivity between users and mobile routers, we need to have constraints in the environments and on the number of robots.

Of course to give an exact threshold on the number of robots necessary to cover an environment is very hard. However, here, we provide an upper safe bound on the number of mobile agents, that is: 


$$
\mathcal{N}_{r} \geq \frac{2 A_{\mathcal{W}}}{\sqrt{3} l_{i j}^{0^{2}}},
$$

where $A_{\mathcal{W}}$ is the area of the workspace where the robots operate and $\sqrt{3} l_{i j}^{0}{ }^{2} / 2$ is the area of the equilateral triangle having for side the length $l_{i j}^{0}$

\section{ALGORITHM DESCRIPTION}

Algorithm 1 illustrates how the simulation scenario has been implemented and summarizes the main events which occur during the simulation.

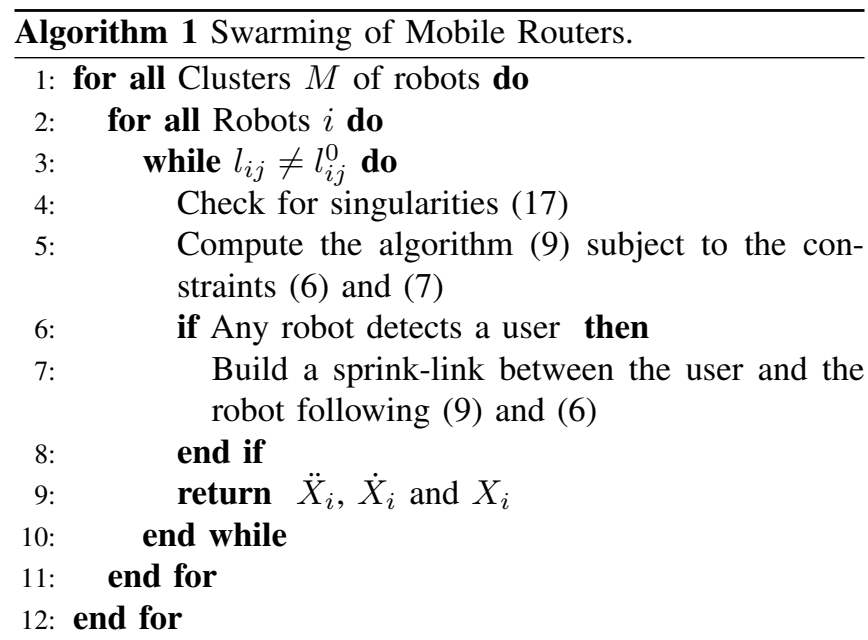

As demonstrated in [22] the computational complexity for each robot is $\mathcal{O}\left(N_{s}^{2}\right)$ where $N_{s}$ is the number of spring-links connected to each robot.

The algorithm stops when the users reach the final positions and don't move and when the spring-mass system reaches equilibrium.

\section{Simulation Results}

In this section we demonstrate the applicability of the developed algorithm through a series of simulations. In particular we analyze two scenarios.

The first simulation in Fig. 2 shows one of the characteristics of the algorithm developed in this paper: the self-healing behavior. The network is able to recognize whenever one or more nodes stop working and can reconfigure to overcome the lacuna.

Fig. 2(a) presents a connectivity graph already built. For simplicity purposes, in this scenario we don't have obstacles and users. The six nodes depicted with a different color in Fig. 2(a) represent robots that simultaneously stop working and thus no longer have sensing capabilities.

In Fig. 2(b), the immediate successive snapshot, we see that the configuration recognizes the "lost robots" and overcomes this situation by building virtual spring links between different mobile routers.

Finally Fig. 2(d), the last step, shows that the configuration reaches a new equilibrium with less nodes in the network.

In the second simulation, Fig. 3, we present a more general scenario in which communication is available in the area under exploration (non-isotropic environment) and to be even

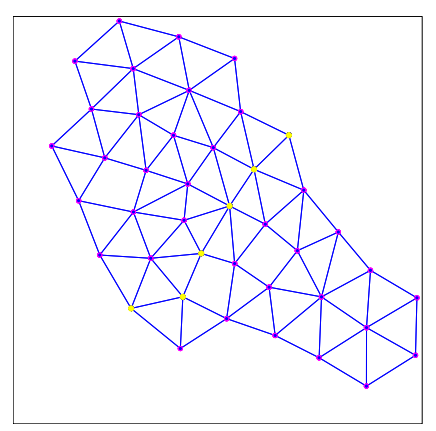

(a)

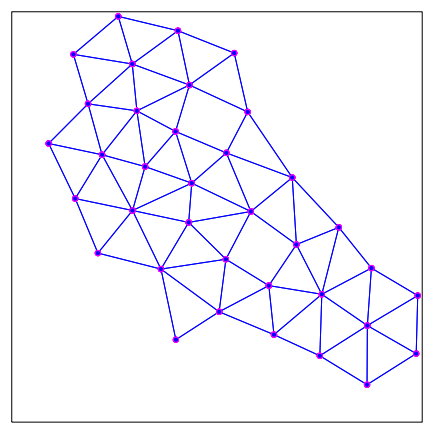

(c)

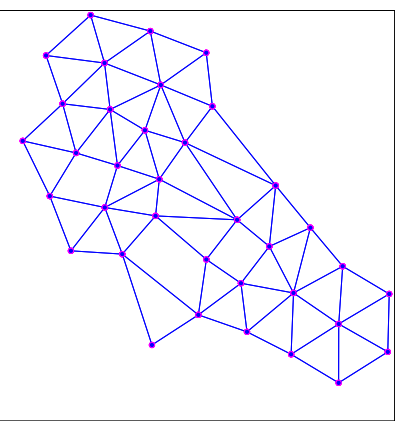

(b)

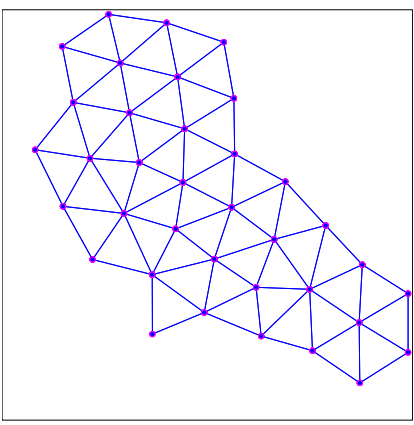

(d)
Fig. 2. Self-healing simulation.

more realistic we constrain communication to certain areas because of the presence of obstacles or disturbances.

The artificial field depicted with concentric patches in Fig. 3 represents a signal that decays toward the center of the patches.

The goal is to keep two users connected with a base station through a network of mobile routers that have the capability to sense the quality of the signal in the environment and therefore adapt to it, moving avoiding obstacles and collisions.

The robots get closer to each other in the center of the potential fields where the communication is poor, while moving far apart where the signal has better quality (Fig. 3(b)(c)). As time progresses (Fig. 3(e)(f)), the robots that are in poor communication positions are attracted to places in the environment with a higher, quality signal, keeping the users connected with a base station and at the same time satisfying the constraints stated in the previous sections.

\section{CONCLUSIONS}

In this work we have presented a framework based on the spring-mass virtual environment and on potential functions. In order to have a uniform distribution of the robots in the environment, we take advantage of the Gabriel Graph in which a mesh of robots is built using switching rules.

Simulation results demonstrate the applicability of the proposed technique: the mesh of routers is able to stretch and keep the users connected with a base station while avoiding obstacles and collisions. The only drawback is that we are able to guarantee connectivity with the users only after the mobile routers detect them or if we introduce boundaries on the environment and on the number of robots.

Future work will be centered on extending the proposed methodology to more realistic situations, including routing 


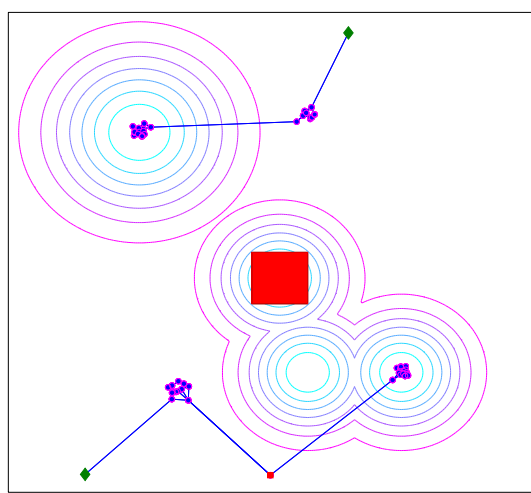

(a)

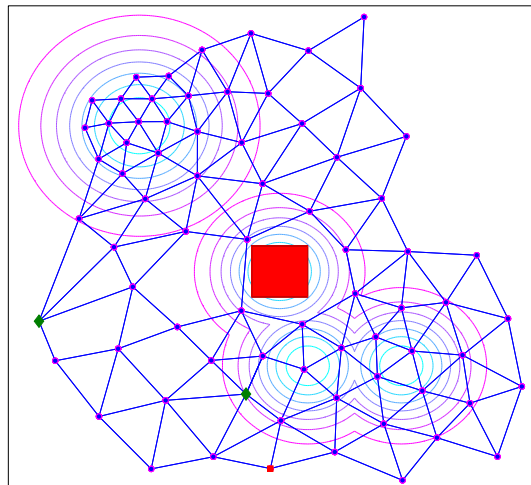

(d)

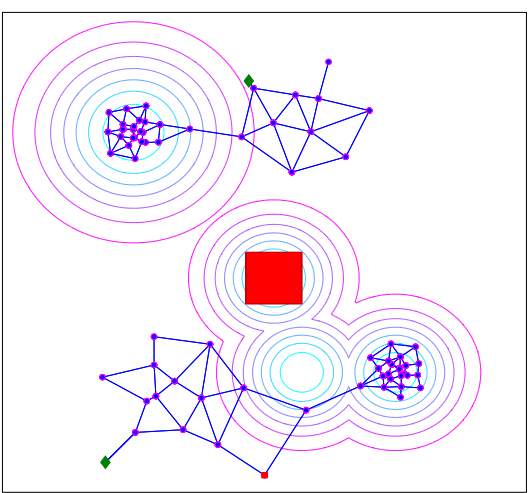

(b)

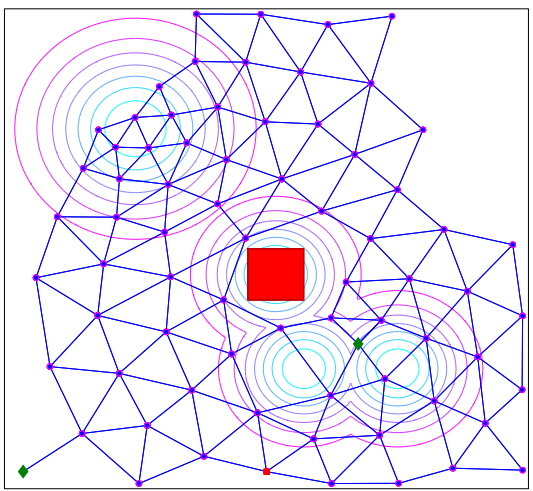

(e)

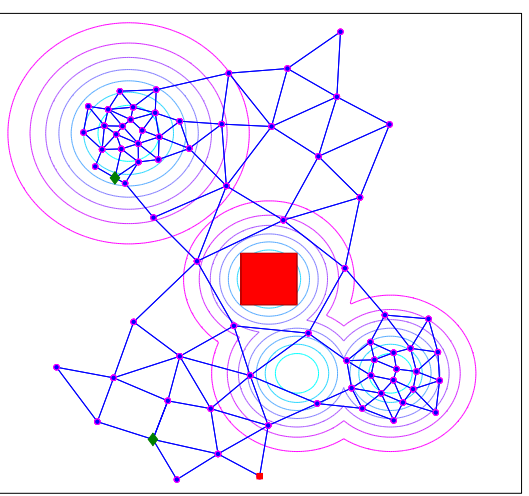

(c)

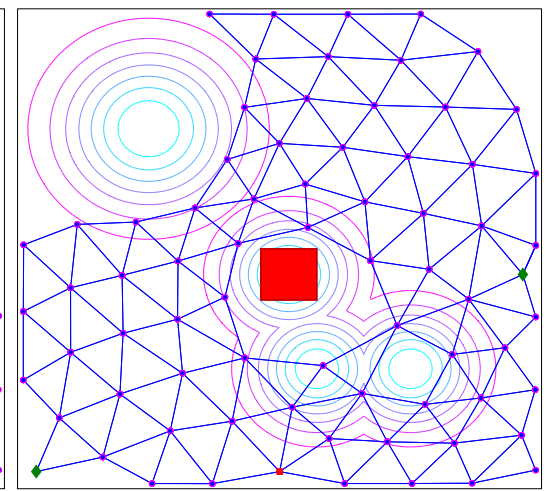

(f)

Fig. 3. Clusters of routers maintaing connectivity between two users (diamonds) and a base station (square on the bottom center) in a non-isotropic environment (concentric circular patches).

and testing the algorithm with experiments in our laboratory. The use of 3-D scenarios with UAV relays is also in our agenda.

\section{ACKNOWLEDGMENTS}

This work is supported by NSF grants ECCS CAREER \#0811347 and IIS \#0812338.

\section{REFERENCES}

[1] A. Goldsmith, Wireless communications. Cambridge Univ Pr, 2005.

[2] N. Bezzo and R. Fierro, "Tethering of mobile router networks," in American Control Conference (ACC), 2010, pp. 6828-6833.

[3] M. McClure, D. R. Corbett, and D. W. Gage, "The DARPA LANdroids program," in Proc. of SPIE, ser. Unmanned Systems Technology XI, G. R. Gerhart, D. W. Gage, and C. M. Shoemaker, Eds., vol. 7332, Orlando, FL, April 2009, pp. 0A-1-0A-10.

[4] M. Lindhé and K. Johansson, "Adaptive Exploitation of Multipath Fading for Mobile Sensors," in IEEE International Conference on Robotics and Automation (ICRA), 2010, pp. 1934-1939.

[5] Y. Mostofi, M. Malmirchegini, and A. Ghaffarkhah, "Estimation of communication signal strength in robotic networks," in IEEE International Conference on Robotics and Automation (ICRA), 2010, pp. 1946-1951.

[6] J. Fink and V. Kumar, "Online methods for radio signal mapping with mobile robots," in IEEE International Conference on Robotics and Automation (ICRA), 2010, pp. 1940-1945.

[7] S. Gil, M. Schwager, B. Julian, and D. Rus, "Optimizing communication in air-ground robot networks using decentralized control," in IEEE International Conference on Robotics and Automation (ICRA), 2010, pp. 1964-1971.

[8] H. Hashemi, "The indoor radio propagation channel," Proceedings of the IEEE, vol. 81, no. 7, pp. 943-968, 1993.

[9] O. Tekdas, P. Plonski, N. Karnad, and V. Isler, "Maintaining Connectivity in Environments with Obstacles," in IEEE International Conference on Robotics and Automation (ICRA), 2010, pp. 19521957.
[10] A. Ghaffarkhah and Y. Mostofi, "Channel Learning and Communication-Aware Motion Planning in Mobile Networks," in American Control Conference (ACC), 2010, pp. 5413-5420.

[11] M. Zavlanos and G. Pappas, "Distributed connectivity control of mobile networks," IEEE Transactions on Robotics, vol. 24, no. 6, pp. 1416-1428, 2008.

[12] S. Anderson, R. Simmons, and D. Golberg, "Maintaining line of sight communications networks between planetary rovers," in IEEE International Conference on Intelligent Robots and Systems (IROS), 2003, pp. 2266-2272.

[13] B. Shucker, T. Murphey, and J. Bennett, "Convergence-Preserving Switching for Topology-Dependent Decentralized Systems," IEEE Transactions on Robotics, vol. 24, no. 6, pp. 1405-1415, 2008.

[14] Y. Kim and M. Mesbahi, "On maximizing the second smallest eigenvalue of a state-dependent graph laplacian," IEEE Transactions on Automatic Control, vol. 51, no. 1, pp. 116-120, 2006.

[15] D. Spears, W. Kerr, and W. Spears, "Fluid-Like Swarms with Predictable Macroscopic Behavior," Lecture Notes in Computer Science, vol. 4324, 2009.

[16] M. Spong, S. Hutchinson, and M. Vidyasagar, Robot modeling and control. Wiley New Jersey, 2006.

[17] T. Rappaport, Wireless communications: principles and practice. Prentice Hall PTR New Jersey, 2002.

[18] F. Bullo, J. Cortes, and S. Martinez, Distributed control of robotic networks: a mathematical approach to motion coordination algorithms. Princeton Univ Pr, 2009.

[19] D. Liberzon, Switching in systems and control. Springer, 2003.

[20] J. Hespanha and A. Morse, "Stability of switched systems with average dwell-time (I)," in IEEE Conference on Decision and Control(CDC), vol. 3, 1999, pp. 2655-2660.

[21] Y. Bhasin and A. Liu, "Bounds for Damping that Guarantee Stability in Mass-Spring Systems," Studies in health technology and informatics, vol. 119, p. 55, 2005.

[22] B. Shucker, "Control of Distributed Robotic Macrosensors," Ph.D. dissertation, Computer Science Department, University of Colorado at Bulder, CO, 2006. 